\title{
Periodic Fever, Aphthous Stomatitis, Pharyngitis, Adenitis Syndrome
}

National Cancer Institute

\section{Source}

National Cancer Institute. Periodic Fever, Aphthous Stomatitis, Pharyngitis, Adenitis

Syndrome. NCl Thesaurus. Code C116917.

An autoinflammatory syndrome of childhood which often resolves in adolescence, and is characterized by periodic fever, aphthous stomatitis, pharyngitis, and cervical adenitis.

The fever cycle generally occurs every three to five weeks, and during the interim periods the child appears healthy; diagnosis is exclusionary. 